\title{
Hepatocyte mitochondrial DNA drives nonalcoholic steatohepatitis by activation of TLR9
}

\author{
Irma Garcia-Martinez, ${ }^{1}$ Nicola Santoro, ${ }^{2}$ Yonglin Chen, ${ }^{1}$ Rafaz Hoque, ${ }^{1}$ Xinshou Ouyang, ${ }^{1}$ Sonia Caprio, ${ }^{2}$ Mark J. Shlomchik, ${ }^{3}$ \\ Robert Lee Coffman, ${ }^{4}$ Albert Candia, ${ }^{4}$ and Wajahat Zafar Mehal ${ }^{1,5}$ \\ 'Department of Internal Medicine and '2Department of Pediatrics, Yale University School of Medicine, New Haven, Connecticut, USA. ${ }^{3}$ Department of Immunology, University of Pittsburgh School of Medicine, \\ Pittsburgh, Pennsylvania, USA. ${ }^{4}$ Dynavax Technologies, Berkeley, California, USA. ${ }^{5}$ Section of Digestive Diseases, Department of Veterans Affairs Connecticut Healthcare, West Haven, Connecticut, USA.
}

\begin{abstract}
Nonalcoholic steatohepatitis (NASH) is the most common liver disease in industrialized countries. NASH is a progressive disease that can lead to cirrhosis, cancer, and death, and there are currently no approved therapies. The development of NASH in animal models requires intact TLR9, but how the TLR9 pathway is activated in NASH is not clear. Our objectives in this study were to identify NASH-associated ligands for TLR9, establish the cellular requirement for TLR9, and evaluate the role of obesity-induced changes in TLR9 pathway activation. We demonstrated that plasma from mice and patients with NASH contains high levels of mitochondrial DNA (mtDNA) and intact mitochondria and has the ability to activate TLR9. Most of the plasma mtDNA was contained in microparticles (MPs) of hepatocyte origin, and removal of these MPs from plasma resulted in a substantial decrease in TLR9 activation capacity. In mice, NASH development in response to a high-fat diet required TLR9 on lysozyme-expressing cells, and a clinically applicable TLR9 antagonist blocked the development of NASH when given prophylactically and therapeutically. These data demonstrate that activation of the TLR9 pathway provides a link between the key metabolic and inflammatory phenotypes in NASH.
\end{abstract}

\section{Introduction}

Hepatocyte steatosis and ballooning and inflammation of the liver constitute steatohepatitis, which occurs in response to a diverse range of stressors ranging from excess alcohol to drug-induced liver injury. Obesity-driven metabolic syndrome is the most common reason for steatohepatitis and is termed nonalcoholic steatohepatitis (NASH) (1). Overnutrition and obesity uniformly result in hepatic steatosis, which can progress to steatohepatitis, fibrosis, and cirrhosis. The biological processes promoting progression from steatosis to steatohepatitis are of great interest but are currently poorly understood.

TLR9 is an endosomal pattern recognition receptor (PRR) for which CpG-rich bacterial DNA and mammalian self-DNA are ligands (2). We have identified a requirement for TLR9 in toxic liver inflammation and fibrosis $(3,4)$, and this has been confirmed for hepatic steatosis and inflammation in the choline-deficient amino acid-defined diet model of NASH (5). Apart from this basic finding of a requirement for TLR9 in the development of NASH, very little is known about the identity of TLR9 ligands and how the TLR9 pathway is activated in NASH. The metabolic changes in NASH suggest that there may be unique interactions with TLR9 biology in NASH, beyond that found for liver injury in general. For example, mitochondrial DNA (mtDNA) has many features that make it a potent ligand for TLR9, and there is an increase in hepatocyte mitochondrial content in NASH (6).

The demonstration of an increase in total liver mtDNA and oxidized DNA (oxDNA) in NASH and the ability of these mole-

Conflict of interest: R.L. Coffman and A. Candia are employees of Dynavax Technologies. Submitted: July 23, 2015; Accepted: December 8, 2015.

Reference information: J Clin Invest. 2016;126(3):859-864. doi:10.1172/JCI83885. cules to activate TLR9 suggest a potential mechanism by which the changes induced by overnutrition result in greater activation of a proinflammatory pathway that leads to a transition from steatosis to steatohepatitis $(6,7)$.

\section{Results and Discussion}

We tested whether hepatocyte nuclear DNA (nDNA) and mtDNA from high-fat diet-induced (HFD-induced) NASH livers are more potent activators of TLR9 than DNA from control livers. Figure 1A shows that mtDNA isolated from hepatocytes of mice fed a HFD for 12 weeks resulted in greater activation of a TLR9 reporter than did mtDNA from mice fed a chow diet (CD). This was confirmed in mouse Kupffer cells (KCs), which demonstrate upregulation of Tnfa transcripts in response to hepatocyte mtDNA from HFD-fed mice (Figure 1B).

Hepatocyte mtDNA is increased in NASH and is known to be proinflammatory (6). For hepatocyte mtDNA to activate TLR9, it would require release from hepatocytes and entry into TLR9containing endosomes of cells capable of mounting an inflammatory response. Plasma extracellular mtDNA levels increase in tissue injuriesinmiceand humansincluding toxicliverinjury (8). Wetested whether a 12-week HFD model of NASH resulted in an increase in plasma DNA. There was an increase in total DNA and mtDNA, but not nDNA in plasma from HFD-fed compared with CD-fed mice (Supplemental Figure 1, A-C; supplemental material available online with this article; doi:10.1172/JCI83885DS1). Plasma from HFD-fed mice gave a small but significantly greater signal than did plasma from CD-fed mice from a TLR9 reporter cell line (Supplemental Figure 1D). To confirm whether these changes were present in humans, plasma from 3 previously well-characterized groups was analyzed: group 1 consisted of lean subjects without 


\section{Table 1. Characteristics of patient groups}

$\begin{array}{lccc} & \begin{array}{c}\text { Group 1: Lean }(n=19) \\ \text { Median (range) }\end{array} & \begin{array}{c}\text { Group 2: Obese, normal ALT ( } \boldsymbol{n}=19) \\ \text { Median (range) }\end{array} & \begin{array}{c}\text { Group 3: Obese, high ALT ( } \boldsymbol{n}=9) \\ \text { Median (range) }\end{array} \\ \text { Age (yr) } & 15.4(9-16.5) & 13.7(9.5-18.8) & 15.1(9.8-17.4) \\ \text { Sex (M/F) } & 11 / 8 & 9 / 10 & 6 / 4 \\ \text { Race (MED/AA/H) } & 9 / 3 / 7 & 6 / 5 / 8 & 3 / 2 / 4 \\ \text { GT (NGT/IGT/T2D) } & 17 / 2 / 0 & 11 / 3 / 1 & 7 / 2 / 0 \\ \text { Z-score BMI } & 0.41(-1.70-1.04) & 2.16(1.75-2.67) & 2.61(1.94-2.82) \\ \text { Fasting glucose (mg/dl) } & 86(77-89) & 87.5(71-96) & 91.5(81-101) \\ \text { 2-h glucose (mg/dl) } & 110(74-138) & 117(92-219) & 125(102-166) \\ \text { Fasting insulin (mcU/ml) } & 14(7-46) & 16(4-109) & 40(20-85) \\ \text { Insulin sensitivity index } & 3.64(2.52-5.53) & 2.91(0.48-8.1) & 1.02(0.51-1.70) \\ \text { ALT (UI/l) } & 13(8-27) & 18(9-21) & 102(81-177)\end{array}$

Demographic, biometric, and metabolic characteristics of the 3 patient groups. Normal ALT levels were below $35 \mathrm{U} / \mathrm{I}$. M, male; F, female; MED, mixed European descent; AA, African-American; H, Hispanic; GT, glucose tolerance; NGT, normal glucose tolerance; IGT, impaired glucose tolerance; T2D, type 2 diabetes.

liver disease and normal alanine transaminase (ALT) levels; group 2 consisted of obese subjects with normal serum ALT levels; and group 3 consisted of obese subjects with elevated ALT levels (Table 1 and ref. 9). We used ALT as a marker of ongoing liver injury. The human data mirrored the mouse HFD model data, with group 3 subjects having increased plasma levels of total DNA and mtDNA, but not nDNA compared with groups 1 and 2 (Figure 1, C-E). In addition, plasma from group 3 subjects produced a stronger signal from the TLR9 reporter cell line (Figure 1F). These data show an increase in plasma mtDNA and greater TLR9 ligand activity in HFD-induced NASH.

Plasma mtDNA could be present in solution, as intact free mitochondria, or as mitochondria enclosed within extracellular microparticles (MPs) (10). Of relevance, in a choline deficiency model of NASH, there is a dramatic increase in MPs of hepatocytic origin in plasma, with hepatocyte MPs visible in the space of Disse (11). To identify whether intact mitochondria were present in plasma from our subjects with NASH and whether they were free or within MPs, we performed flow cytometric analysis of plasma from subjects in each of the 3 groups. Mitochondria were readily detectable in plasma by flow cytometry after staining with MitoTracker Deep Red, which stains mitochondria with an intact membrane potential, and MPs were identified by a combination of size gating $(0.2-1.0 \mu \mathrm{m})$ and positivity for the plasma membrane dye PKH67 green. Dual staining with MitoTracker Deep Red and PKH67 allows the identification of mitochondria enclosed within a plasma membrane (Figure 1G, upper right quadrant). Obese patients with elevated serum ALT levels had a greater percentage of mitochondria inside MPs compared with lean subjects (Figure $1 \mathrm{H}$ ), and there was an increase in the percentage of MPs containing mitochondria (Figure 1I), collectively demonstrating a relative increase in MPs containing mitochondria. MP proteins were probed with an $\mathrm{Ab}$ specific to the hepatocyte protein arginase 1 (Arg1), which gave a strong positive signal, while the same preparations did not contain the platelet integrin CD41 (Figure 1J). mtDNA is at higher risk of oxidation, which is known to increase the ability of DNA to activate TLR9 (12). We quantified the percentage of MPs containing oxDNA by detecting $8-\mathrm{OH}$-deoxyguanosine $(8-\mathrm{OH}-\mathrm{dG})$ and found that there was a significant increase in oxDNA in MPs in plasma from obese subjects with elevated serum ALT levels (Figure 1K). Interestingly, there was no increase in the percentage of free mitochondria staining for oxDNA, and the cellular origin of these free mitochondria is yet to be determined. The hepatocyte origin of plasma MPs containing mitochondria with increased oxDNA is consistent with the findings of increased oxidation of hepatocyte DNA in NASH (6). Finally, we confirmed that MPs were responsible for the ability of plasma to activate a TLR9 reporter cell line, as the majority of this activity was lost in MP-free plasma (Figure 1L).

TLR9 ligands can preferentially activate 2 downstream signaling pathways, resulting in differing degrees of production of NF- $\kappa \mathrm{B}$-dependent proinflammatory cytokines and IRF7-dependent type 1 IFN (13). The IRF7-dependent pathway can upregulate the IL-1 receptor antagonist (IL-1RA), which has antiinflammatory effects in the liver (14). To test whether mtDNA from hepatocytes of HFD-fed mice can activate type 1 IFN and IL-1RA, primary murine macrophages were stimulated with nDNA and mtDNA from hepatocytes of HFD-fed and CD-fed mice, and mRNA levels of Ifna, Ifnb, and Il1ra were quantified (Supplemental Figure $2, \mathrm{~A}-\mathrm{C}$ ). In contrast to the upregulation of TNF- $\alpha$, hepatocyte mtDNA from HFD-fed mice, when compared with mtDNA from CD-fed mice, resulted in no change in Ifna, Ifnb, or Illra levels (15). This demonstrates that mtDNA from hepatocytes of HFDfed mice results in the selective upregulation of proinflammatory cytokines, but not type 1 IFN.

Total body TLR9 deletion results in loss of NASH in experimental models, but the cellular requirement for TLR9 is not known (5). Liver macrophages, which include resident KCs and infiltrating monocytes, are the main proinflammatory cell populations in NASH. We tested the role of TLR9 signaling in these cells by generating mice in which TLR9 was removed from lysozyme-producing cells. $\operatorname{Tl} \mathrm{f}^{f / f l} \mathrm{fl}$ homozygous and lysozyme-Cre heterozygous (Lysm-Cre Tlr $9^{f / f l}$ ) mice were generated, and along with total TLR9-deficient mice, were placed on a HFD. Lysm-Cre $\operatorname{Tl} 9^{f / f l}$ mice had a reduction in TLR9 expression in neutrophils and KCs (Supplemental Figure 3, A-C). WT mice on a HFD for 12 weeks developed significant hepatic steatosis, ballooning, inflam- 

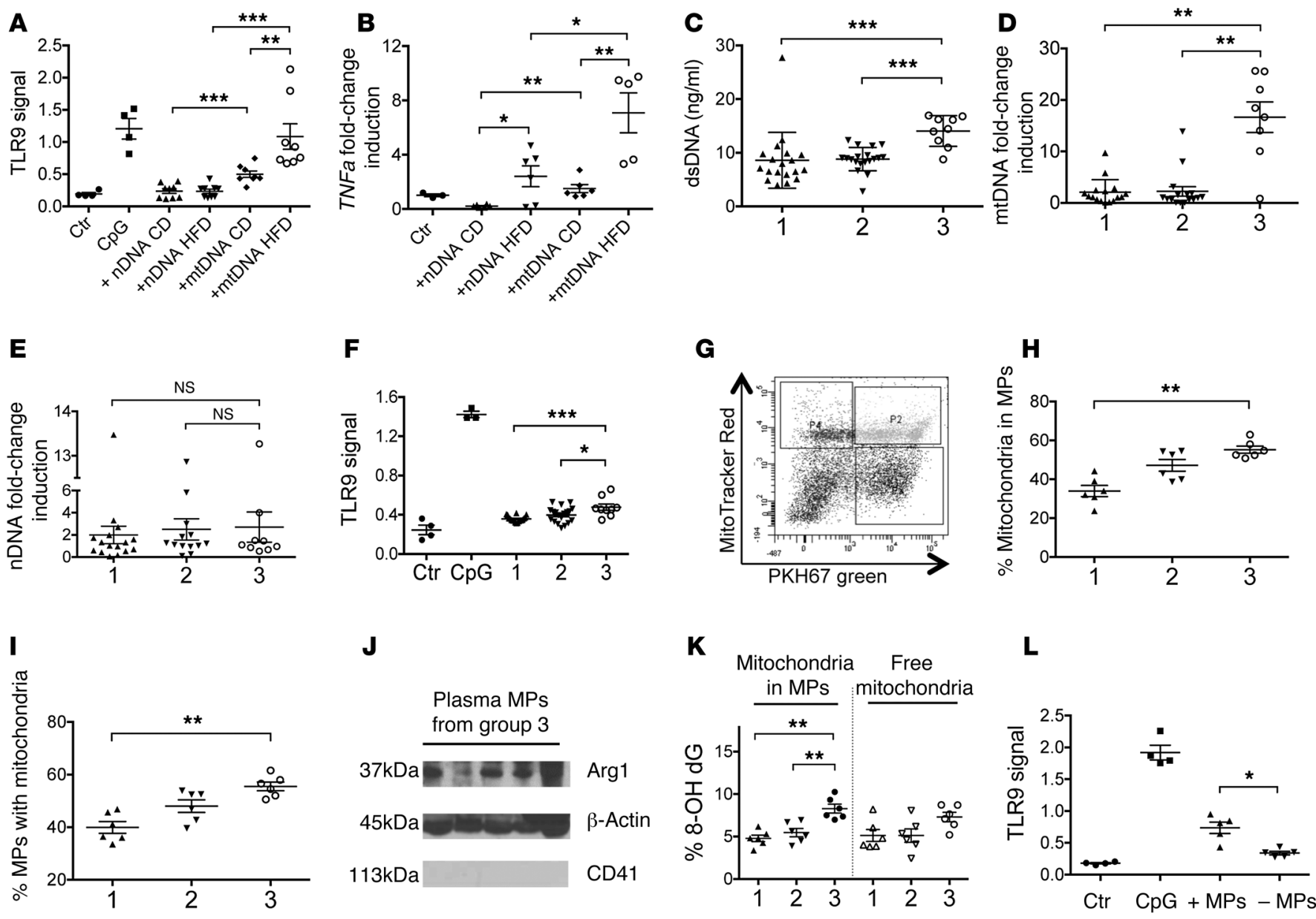

Figure 1. Hepatocyte mtDNA in NASH has increased oxidation and is present in plasma MPs. Soluble nDNA and mtDNA from hepatocytes from CD-fed $(n=9)$ and HFD-fed $(n=9)$ mice were added to TLR9 HEK reporter cell lines (A) and murine KCs (CD, $n=6 ; H F D, n=6)$ (B) for 12 and 8 hours, respectively, and the upregulation of TLR9 signal and Tnfa message was quantified. Human plasma: group 1: lean subjects $(n=19)$; group 2: obese subjects with normal serum ALT levels $(n=19)$; and group 3: obese subjects with elevated serum ALT levels $(n=9)$. Group 3 plasma had more total DNA (C) and mtDNA (D), but not nDNA (E), and a greater ability to activate a TLR9 reporter cell line (F). MPs from group $1(n=6)$, group $2(n=6)$, and group $3(n=6)$ were prepared by high-speed centrifugation of platelet-free plasma after PKH67 plasma membrane staining ( $x$ axis) and MitoTracker Deep Red staining (viable mitochondria, $y$ axis). Mitochondria within MPs are shown in the top right quadrant (C). Gating on mitochondria shows that in group 3, a greater percentage was inside MPs (H), and gating on MPs shows that in group 3, there was an increase in the percentage of MPs that contained mitochondria (I). Protein was isolated from MPs from group 3 subjects and examined by Abs specific to the hepatocyte plasma membrane protein Arg1 or platelet integrin CD41 ( $n=2$ ) (J). After gating on MPs (gate size, 0.2-1 mM and PKH67+) and staining for oxDNA, an increase in oxidation status of mtDNA was evident in plasma MPs from group 3 subjects (K). MPs were removed from plasma by centrifugation, which resulted in a reduction of the ability of human plasma to activate TLR9 reporter cell lines $(\mathbf{L})$. Data represent the mean \pm SEM. ${ }^{*} P<0.05,{ }^{* *} P<0.01$, and ${ }^{* * *} P<0.005$, by Mann-Whitney $U$ test. Ctr, control.

mation, and elevated transaminases, and these were all significantly less severe in Tlr9-KO and Lysm-Cre Tlr $9^{f / f l}$ mice (Figure 2, A-C). Tlr9-KO and Lysm-Cre Tlr9f/ll mice also had reduced upregulation of total liver inflammatory cytokine gene expression while on a HFD (Figure 2D) and no difference in food intake (Supplemental Figure 3D). The reduction in hepatocyte steatosis in $\mathrm{Lysm}$-Cre $\mathrm{Tlr}^{\mathrm{f} / \mathrm{l} / \mathrm{l}}$ mice is interesting and was also found in an earlier model of methionine choline-deficient (MCD) diet steatosis in Tlr $9^{-/}$mice (5). The ability of IL-1 $\beta$ to induce hepatocyte steatosis suggests a direct role of proinflammatory cytokines. As lysozymes are expressed by neutrophils, monocytes, and tissue macrophages, this approach is not exclusive to liver macrophages and KCs but provides significant support for their involvement. To further confirm that proinflammatory KC activation occurs in NASH, NF- $\mathrm{kB}$ activation was examined in KCs in vivo using an NF- $\mathrm{KB}$ reporter mouse and gating on CD11b F4/80 doublepositive cells. The HFD induced upregulation of the NF- $\mathrm{KB}$ reporter in CD11b F4/80 double-positive cells (Supplemental Figure 3E), and KCs from WT mice on a HFD had greater proinflammatory responsiveness to a TLR9 ligand (Supplemental Figure 3, F-H).

The demonstration of high plasma TLR9 ligand activity and a requirement for TLR9 in the development of HFD-induced NASH have immediate therapeutic implications. TLR9 antagonists have excellent safety profiles and represent a novel therapeutic strategy for NASH. We tested whether the TLR7/9 antagonist IRS954 could block the ability of hepatocyte mtDNA from HFD-fed mice to activate proinflammatory cytokines in primary macrophages. WT mice were placed on a HFD and concurrently administered 
A

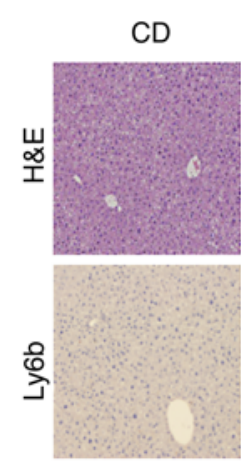

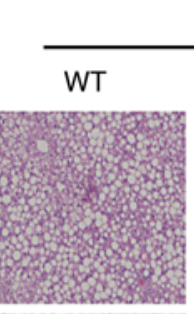

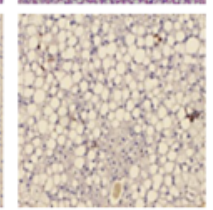

HFD

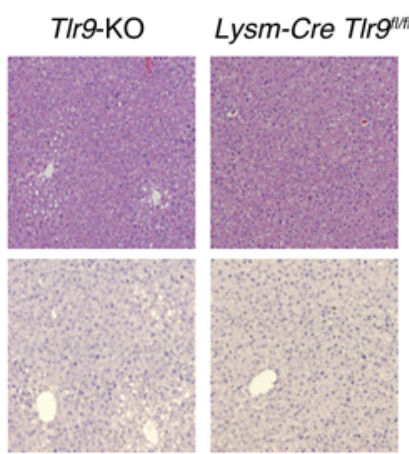

B

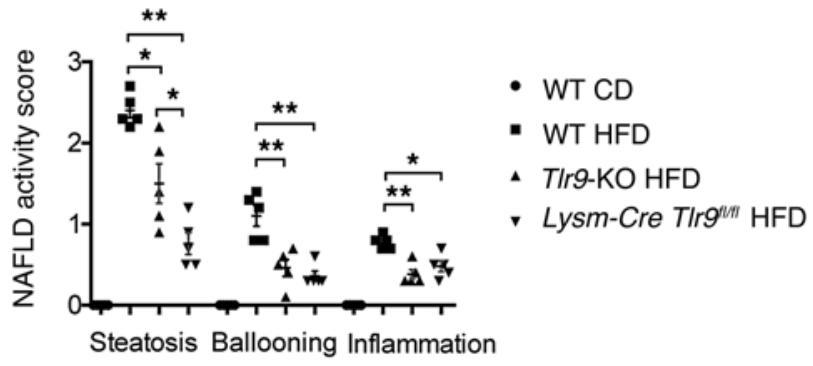

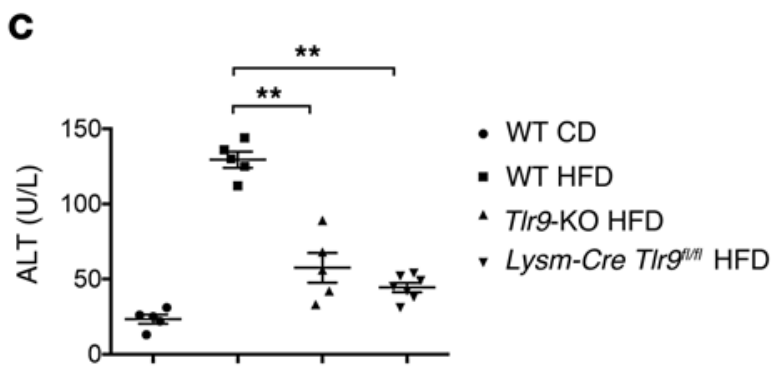

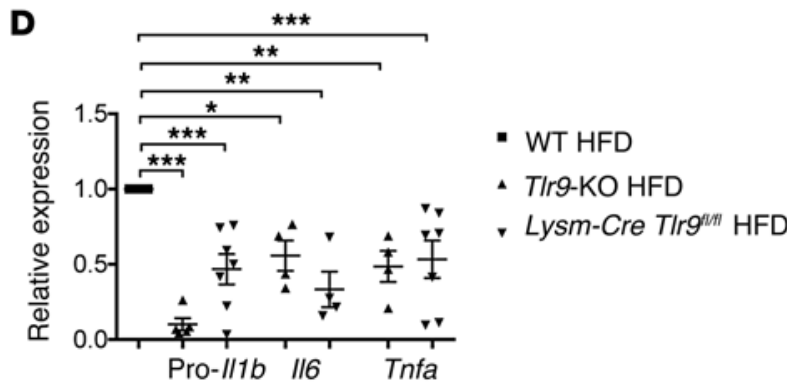

Figure 2. Total and lysozyme-expressing cell-specific loss of TLR9 protects against NASH. WT ( $n=5)$, TLR9-deficient (T/rg-KO, $n=5)$, and T/rgfl/fl homozygous and lysozyme-Cre heterozygous (Lysm-Cre T/rgfl/fl,$n=7$ ) mice were placed on a HFD for 12 weeks, and liver histology (steatosis, ballooning, and inflammation) was quantified (A and B). For total and lysosome-expressing cell-specific deletion of TLR9, there was a significant reduction in serum ALT (C) and reduced upregulation of inflammatory cytokines in whole liver (D) when compared with WT mice on a HFD. Original magnification, $\times 100$. Data represent the mean \pm SEM. ${ }^{*} P<0.05,{ }^{*} P<0.01$, and ${ }^{* * *} P<0.005$, by Mann-Whitney $U$ test.

IRS954 (s.c., $5 \mathrm{mg} / \mathrm{kg}$ weekly). This resulted in a significant reduction in steatosis, ballooning and inflammation, serum transaminases, and inflammatory cytokine transcript levels (Figure 3, A-D), with no difference in food intake (Supplemental Figure 4). A therapeutic protocol calling for initiation of IRS954 after 8 weeks of a HFD, up to a total of 12 weeks on a HFD, also resulted in a significant reduction in hepatic steatosis, ballooning and inflammation, and serum transaminases, again, with no difference in food intake (Supplemental Figure 5). Our data also open up the possibility of TLR9 ligand-focused therapies such as removal of plasma mitochondria and MPs using Ab-mediated strategies.

This provides what we believe to be a novel link between the metabolic changes associated with steatosis (mitochondrial ROS, oxidized mtDNA) and the development of a proinflammatory response via TLR9 activation. Hepatocyte mtDNA from NASH livers has a greater ability to activate TLR9, and increased amounts of plasma MPs of hepatocyte origin containing intact mitochondria with greater amounts of oxDNA result in greater activation of TLR9 and a skewed proinflammatory response. This identifies a number of steps where this process can be therapeutically targeted, including mtDNA oxidation, MP release, MP uptake, and TLR9 ligand binding.

A number of mechanisms limit TLR9 responsiveness to selfDNA including low numbers of CpG repeats in mammalian DNA and sensitivity of self-DNA to DNase II. These do not apply to mtDNA, which is relatively $\mathrm{CpG}$ rich and is protected from DNase II by the mitochondrial membranes. Mitochondria also contain
HMGB1, which can enhance TLR9 signaling by DNA, and additional damage-associated molecular patterns (DAMPs), such as formyl peptides $(16,17)$. The systemic distribution of hepatocytederived mitochondria with the ability to activate TLR9 may have consequences in addition to hepatic inflammation. A chronic, low-level increase in plasma TLR9 ligand activity can be expected to activate TLR9 systemically, and this is consistent with the low-level systemic inflammation in NASH that is found in many organs including white adipose tissues and its loss in the absence of TLR9 (5). This leads to the intriguing possibility that hepatocyte-derived mtDNA is responsible for activation of macrophage populations in extrahepatic sites.

\section{Methods}

Statistics. Differences between 2 groups were compared with a Mann-Whitney $U$ test using GraphPad Prism 6 software (GraphPad Software). A $P$ value of less than 0.05 was considered significant. See the Supplemental Methods for additional details.

Study approval. This study was approved by the Yale University Animal Care and Use Committee and the Human Investigation Committee.

\section{Author contributions}

IGM, NS, YC, RH, and XO performed experimental work, data analysis, and reviewed the manuscript. RLC, AC, and MJS were responsible for experimental design, data analysis, and review of the manuscript. SC handled clinical management and phenotypic characterization of subjects. IGM and WZM were responsible for experimental design, data analysis, and manuscript preparation. 
A

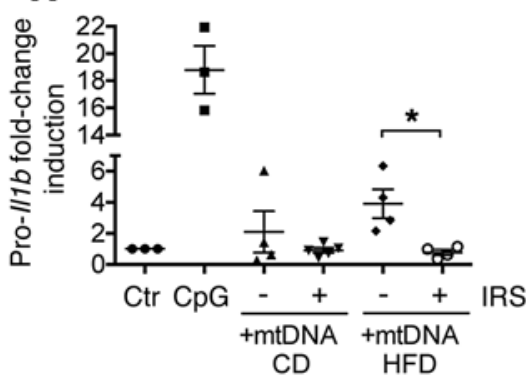

C

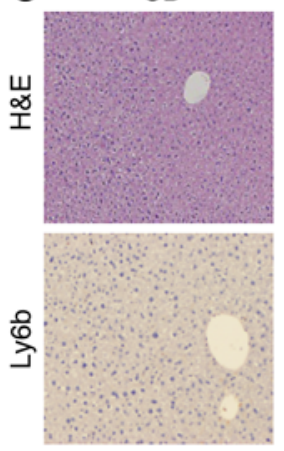

E

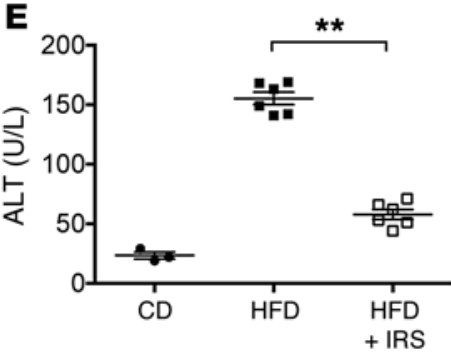

B

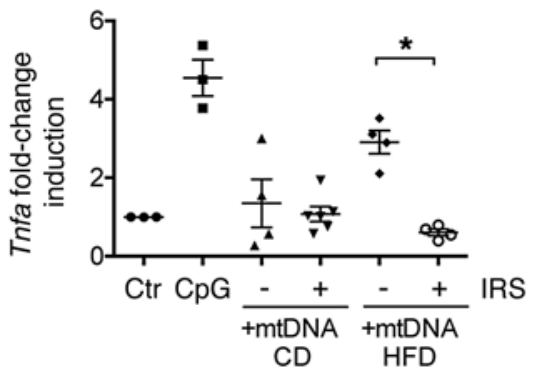

$\mathrm{HFD}+\mathrm{IRS}$

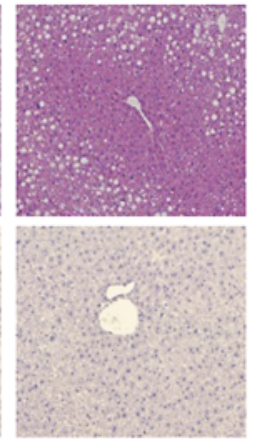

D
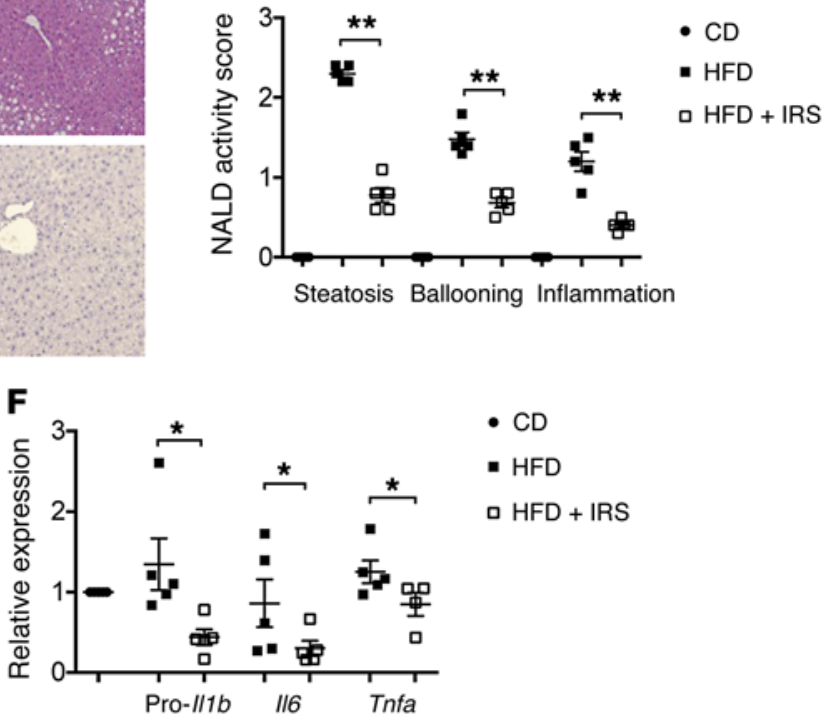

Figure 3. Weekly administration of a TLR9 antagonist protects against NASH. Soluble mtDNA ( $100 \mathrm{ng} / \mathrm{ml})$ from hepatocytes of CD- and HFD-fed mice was added to KCs in the presence and absence of IRS954 (IRS) for 8 hours, and the upregulation of pro-I/1b and Tnfa message was quantified (A and B). In a HFD model of NASH, weekly s.c. administration of the TLR9 antagonist IRS954 (5 mg/kg, $n=5$ ) resulted in reduced steatosis, ballooning, and inflammation (C and $\mathbf{D})$, reduced ALT $(\mathbf{E})$, and reduced upregulation of inflammatory cytokines in the liver $(\mathbf{F})$ compared with mice fed a HFD without injection of IRS954 $(n=5)$. Original magnification, $\times 100$. Data represent the mean \pm SEM. ${ }^{*} P<0.05$ and ${ }^{* *} P<0.01$, by Mann-Whitney $U$ test.

\section{Acknowledgments}

This study was funded by NIH grants 2R56DK076674-06 and 5U01AA021912-02 (to W. Zafar Mehal); K08DK092281 (to R. Hoque); 2R42DK101221-1 (to A. Candia); and 2P01AR050256 (awarded to Ann Marshak-Rothstein and subcontracted to M.J. Shlomchik). This study was also funded by VA Merit awards to W. Zafar Mehal. The Yale Liver Center core facilities were funded by NIH grant DK P3O-034989. We thank C. Jobin for the NF-kB reporter mice and R. Flavell for the Tlr9-KO mice.

Address correspondence to: Wajahat Zafar Mehal, Section of Digestive Diseases, Yale University, 1080 Laboratory of Medicine and Pediatrics, P.O. Box 208019, New Haven, Connecticut 065208019, USA. Phone:203.785.3411; E-mail:wajahat.mehal@yale.edu.
1. Rinella ME, Sanyal AJ. NAFLD in 2014: Genetics, diagnostics and therapeutic advances in NAFLD. Nat Rev Gastroenterol Hepatol. 2015;12(2):65-66.

2. Leadbetter EA, Rifkin IR, Hohlbaum AM, Beaudette BC, Shlomchik MJ, Marshak-Rothstein A. Chromatin-IgG complexes activate B cells by dual engagement of IgM and Toll-like receptors. Nature. 2002;416(6881):603-607.

3. Imaeda AB, et al. Acetaminophen-induced hepatotoxicity in mice is dependent on Tlr9 and the Nalp3 inflammasome. JClin Invest. 2009;119(2):305-314.
4. Watanabe A, et al. Apoptotic hepatocyte DNA inhibits hepatic stellate cell chemotaxis via toll-like receptor 9. Hepatology. 2007;46(5):1509-1518.

5. Miura K, et al. Toll-like receptor 9 promotes steatohepatitis by induction of interleukin-1 $\beta$ in mice. Gastroenterology. 2010;139(1):323-334.

6. Koliaki C, et al. Adaptation of hepatic mitochondrial function in humans with non-alcoholic Fatty liver is lost in steatohepatitis. Cell Metab. 2015;21(5):739-746.

7. Shimada K, et al. Oxidized mitochondrial DNA activates the NLRP3 inflammasome during apoptosis. Immunity. 2012;36(3):401-414.

8. Zhang Q, et al. Circulating mitochondrial DAMPs cause inflammatory responses to injury. Nature. 2010;464(7285):104-107.

9. Santoro $\mathrm{N}$, et al. The association between hepatic fat content and liver injury in obese children and adolescents: effects of ethnicity, insulin resistance, and common gene variants. Diabetes Care. 2013;36(5):1353-1360.

10. Boudreau LH, et al. Platelets release mitochondria serving as substrate for bactericidal group 
IIA-secreted phospholipase A2 to promote inflammation. Blood. 2014;124(14):2173-2183.

11. Povero D, et al. Circulating extracellular vesicles with specific proteome and liver microRNAs are potential biomarkers for liver injury in experimental fatty liver disease. PLoS One. 2014;9(12):e113651.

12. Yoshida H, Nishikawa M, Kiyota T, Toyota H, Takakura Y. Increase in CpG DNA-induced inflammatory responses by DNA oxidation in macrophages and mice. Free Radic Biol Med.
2011;51(2):424-431.

13. Sasai M, Linehan MM, Iwasaki A. Bifurcation of Toll-like receptor 9 signaling by adaptor protein 3. Science. 2010;329(5998):1530-1534.

14. Petrasek J, Dolganiuc A, Csak T, Kurt-Jones EA, Szabo G. Type I interferons protect from Toll-like receptor 9-associated liver injury and regulate IL-1 receptor antagonist in mice. Gastroenterology. 2011;140(2):697-708.

15. Tosello-Trampont AC, Landes SG, Nguyen V, Novobrantseva TI, Hahn YS. Kuppfer cells trig- ger nonalcoholic steatohepatitis development in diet-induced mouse model through tumor necrosis factor-alpha production. J Biol Chem. 2012;287(48):40161-40172.

16. Ito $\mathrm{H}$, et al. HMGB1 facilitates repair of mitochondrial DNA damage and extends the lifespan of mutant ataxin-1 knock-in mice. EMBO Mol Med. 2015;7(1):78-101.

17. Ivanov $S$, et al. A novel role for HMGB1 in TLR9mediated inflammatory responses to CpG-DNA. Blood. 2007;110(6):1970-1981. 